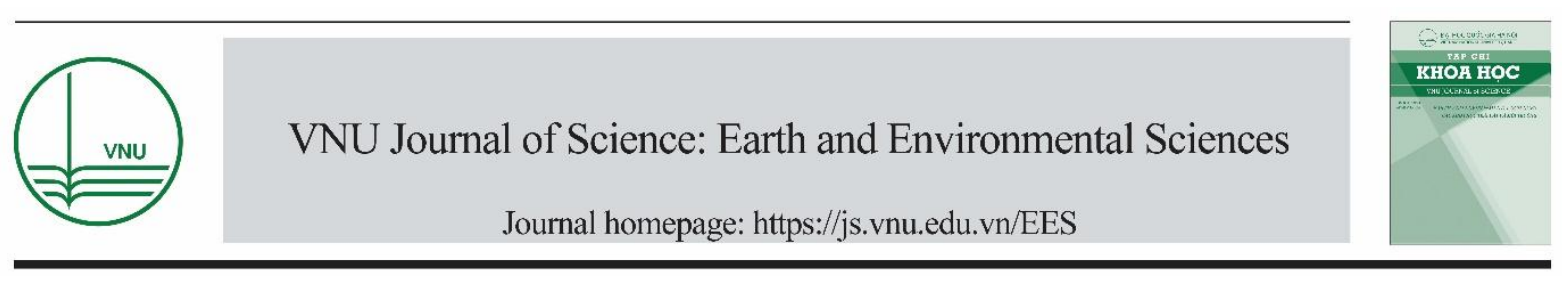

\title{
Original Article \\ Analyzing the Displacement of Horizon Geodetic Network at Tuyen Quang Hydropower
}

\author{
Dinh Xuan Vinh* \\ Hanoi University of Natural Resources and Environment, \\ 41A Phu Dien, Cau Dien, Tu Liem, Hanoi, Vietnam
}

Received 27 May 2019

Revised 16 July 2019; Accepted 02 August 2019

\begin{abstract}
The world mathematicians given many method to adjust the free network, in which the confirmation that the first norm of the solution vectors must minimizing to be the standard for finding the solution in a multitude of solutions. This also conform with the weight transformation process in the deformation model to find the solution for the most probable model, developed by Adam Chrzanowski. The geodetic base point at hydropower plants are used as benchmarks to assess the displacement of test points are attached on the dam. This article presents the iterative weight transformation technique of the problem handle the free geodetic network at Tuyen Quang hydropower. The results showed that the largest displacement value was $2.2 \mathrm{~mm} /$ year and equivalent to the actual measurement error. This calculation method provides more useful information about the displacement model of geodetic base points, helping to plan a large-scale project safety assurance.
\end{abstract}

Keywords: Displacement, Minimizing the first norm of vectors, Geodetic base points.

\footnotetext{
* Corresponding author.

E-mail address: dxvinh@hunre.edu.vn

https://doi.org/10.25073/2588-1094/vnuees.4398
} 


\title{
Phân tích biến dạng lưới mặt bằng tại thủy điện Tuyên Quang
}

\author{
Đinh Xuân Vinh* \\ Truò̀ng Đại học Tài nguyên và Môi truòng Hà Nội, 41A Phú Diễn, Cầu Diễn, Tù Liêm, Hà Nội, Việt Nam \\ Nhận ngày 27 tháng 5 năm 2019 \\ Chỉnh sửa ngày 16 tháng 7 năm 2019; Chấp nhận đăng ngày 02 tháng 8 năm 2019
}

\begin{abstract}
Tóm tắt: Bình sai lưới tự do được các nhà toán học thế giới đưa ra nhiều phương pháp giải, trong đó xác nhận Chuẩn bậc nhất của vector nghiệm phải nhỏ nhất làm tiêu chuẩn để tìm lời giải cho bài toán vô số nghiệm. Điều này cũng trùng hợp với quá trình biến đổi trọng số trong mô hình biến dạng để tìm lời giải cho mô hình xác suất nhất, do Adam Chrzanowski phát triển. Các điểm cơ sở trắc địa tại công trình thủy điện được sử dụng như những điểm chuẩn để đánh giá sự chuyển dịch của các điểm kiểm tra gắn trên thân đập ngắn nước. Bài báo này trình bày kỹ thuật tính chuyển dịch của các điểm cơ sở trắc địa tại thủy điện Tuyên Quang. Kết quả cho thấy giá trị chuyển dịch lớn nhất tương đương sai số đo đạc thực tế. Phương pháp tính này cung cấp thêm góc nhìn mới về mô hình dịch chuyển của các điểm cơ sở trắc địa, giúp hoạch định phương án đảm bảo an toàn công trình sau này.

Tù khoá: Chuyển dịch, Cực tiểu hoá chuẩn bậc nhất vector, Điểm cơ sở trắc địa.
\end{abstract}

\section{Mở đầu}

Phân tích biến dạng là một phần của công tác trắc địa, nhưng quá trình này liên quan tới một mô hình toán - lý phức tạp. Nếu chỉ xét riêng biến dạng hình học, việc xác định các vector biến dạng được thực hiện dựa trên các bước Nhận dạng mô hình - Ước lượng mô hình - Đánh giá mô hình [1]. Quan trắc biến dạng có tầm quan trọng lớn trong nhiều hoạt động liên quan đến kỹ thuật khảo sát. Các công trình xây dựng cần được theo dõi trong suốt thời gian xây dựng và sử dụng của chúng; các hoạt động của con người cũng là nguyên nhân gây ra chuyển dịch trên bề mặt trái đất, ví dụ như lún do khai thác mỏ, khai thác dầu hoặc nước ngầm, xây dựng các hồ chứa lớn. Với tiến bộ kỹ thuật hiện nay, cùng với biến động về môi trường và hiện tượng biến đổi khí hậu, mối quan tâm trong nghiên cứu về chuyển dịch vỏ trái đất ngày càng tăng. Từ đó, yêu cầu nâng cao độ chính xác và độ tin cậy trong đánh giá ổn định điểm khống chế trắc địa là đòi hỏi bức thiết.

Về cơ bản, có cả lý do thực tế và lý do khoa học cho việc nghiên cứu biến dạng. Lý do thực tế là kiểm tra sự ổn định của các cấu trúc địa chất, kết cấu công trình và thiết bị cơ khí, đánh giá mức độ nguy hiểm của tình trạng bất ổn định, phát hiện các yếu tố ban đầu của một rủi ro. Lý do khoa học đó là sự cần thiết để hiểu rõ hơn cơ

\footnotetext{
* Corresponding author.

E-mail address: dxvinh@hunre.edu.vn
}

https://doi.org/10.25073/2588-1094/vnuees.4398 
chế của biến dạng, kiểm tra các lý thuyết mới bao gồm cả các thiết kế trong xây dựng công trình [2]. Từ đó thiết lập các phương pháp dự báo an toàn.

Việc phân tích biến dạng thường phải đối phó với lượng biến dạng rất nhỏ, thậm chí tương đương với sai số của phương pháp đo. Do đó, phân tích phải cực kỳ cẩn thận để đưa ra quyết định đúng đắn về mô hình biến dạng của cấu trúc [1].

Vào năm 1978, Hội nghị các nhà Khảo sát quốc tế (FIG) đã thành lập Uy ban 6 chuyên trách Phân tích biến dạng do giáo sư Chrzanowski là chủ tịch. Nhiệm vụ chính của Ủy ban 6 là: $1 /$ Tối ưu hóa thiết kế mạng lưới quan trắc; 2 / Đánh giá dữ liệu quan trắc, xác nhận trị đo thô và sai số hệ thống; 3/ Phân tích biến dạng hình học; 4/ Giải thích ý nghĩa vật lý của biến dạng [3].

Trong khoảng thời gian từ đó đến nay, các nhóm của Ủy ban 6 tại các trung tâm nghiên cứu nhu: Delft, Fredericton, Hannover, Karlsruhe, Munich đã công bố nhiều thành quả về phương pháp quan trắc, phân tích và xử lý số liệu biến dạng [3]. Đặc biệt, các phương pháp phân tích biến dạng được Ủy ban 6 công bố mang tính tổng hợp, kế thừa và phát huy.

Một số phương pháp đã dùng trước đây [1] như: Phương pháp Kostekhel, sử dụng sai số giới hạn của kết quả thống kê tọa độ điểm quan trắc làm thước đo sự ổn định của mốc trắc địa. Mốc được chọn làm điểm khởi tính phải nhận được kết quả $[\mathrm{pvv}]=\min$; Phương pháp Trernhikov, sử dụng nguyên lý "Tọa độ trung bình của lưới không đổi trong thời gian quan trắc"; Phương pháp "Phân tích tương quan", sử dụng độ lệch chuẩn trên mỗi số liệu đo để phân tích tương quan giữa các thời điểm quan trắc và đánh giá chất lượng số liệu đo; Phương pháp "Mô hình toán học", sử dụng điều kiện phụ kèm bình sai lưới tự do, sau đó kiểm tra sai số giới hạn của tọa độ các điểm sau bình sai, nếu sai số giới hạn lớn hơn 3 lần sai số trung phương thì cho rằng điểm tọa độ đó không ổn định. Phương pháp "Bình sai lưới tự do", sử dụng phương pháp tính nhích dần điều kiện phụ trong bình sai lưới tự do và hệ số giới hạn của độ lệch chuẩn trong thống kê toán học để đánh giá điểm trắc địa bất ổn định.
Trên cơ sở nhiệm vụ của Ủy ban 6, trung tâm nghiên cứu thuộc Đại học Delft do Kok lãnh đạo đã đề xuất [2] phương pháp phân tích độ ổn định của điểm quan trắc dựa trên lý thuyết loại trừ sai số thô của Baarda. Đặc điểm chính của phương pháp là kiểm định thống kê toán học tính thống nhất về cấu trúc hình học của mạng lưới. Nếu kiểm định thất bại, sử dụng phương pháp thử để xác định điểm bất ổn định.

Trung tâm nghiên cứu thuộc Đại học Bonn do Koch đề xuất [2] cũng tương tự như phương pháp của Đại học Delft, nhưng phương pháp phát hiện điểm bất ổn định có khác. Trước tiên, từ trường chuyển dịch và elip sai số của các điểm trong lưới có được sau bình sai lưới tự do, tìm ra những điểm ổn định nhất, dùng chúng để xác định hệ thống lưới mới. Đây là một quá trình tính lặp sử dụng phép biến đổi $\mathrm{S}$ cộng trọng số, với trọng số của điểm ổn định được gán giá trị 1 , các điểm khác gán giá trị 0 . Quá trình tính lặp dừng khi tất cả các điểm ổn định đều được dùng để xác định hệ thống lưới mới.

Phương pháp của trung tâm nghiên cứu thuộc Đại học Hannover chủ yếu do Pelzer và Niemier đề xuất [2]. Tư tưởng của phương pháp là: Tiến hành kiểm định tính thống nhất của hai chu kỳ quan trắc. Nếu kiểm định tổng thể này được thông qua, các điểm trắc địa đều ổn định. Nếu không được thông qua, phương pháp tìm điểm bất ổn định là phương pháp thử. Tuần tự bỏ đi một điểm và tính mức độ giảm thiểu của tính thống nhất cấu hình lưới. Điểm nào làm cho tính thống nhất đó giảm thiểu nhiều nhất tức là điểm bất ổn định. Sau khi loại trừ điểm bất ổn định, lặp lại quá trình trên cho đến khi tính thống nhất của cấu hình lưới được thông qua thì dừng.

Phương pháp "Tuần tự thay thế xác định dần trọng số” do trung tâm nghiên cứu thuộc Đại học Fredericton chủ yếu do Adam Chrzanowski và Chen Yongqi đề xuất $[2,9]$. Nội dung chủ yếu của phương pháp đề cập tới tối thiểu hóa chuẩn bậc nhất của vector chuyển dịch, từ đó xác lập một hệ thống lưới lý tưởng làm cho trường chuyển dịch ít bị méo mó nhất, có lợi cho việc sơ bộ phát hiện mô hình biến dạng. Quá trình tính toán trọng số cho các điểm trong lưới phải tính 
lặp mấy lần để tối thiểu hóa được vector biến dạng. Sau đó xác định được giá trị biến dạng chân thực nhất.

Liên quan tới quá trình xác định điểm ổn định trong lưới trắc địa, thuật toán bình sai lưới tự do cũng được sử dụng. Trước tiên, lưới tự do được định nghĩa như là một mạng lưới thiếu yếu tố xác định trong không gian. Cẩu trúc của lưới được xác định thông qua các trị đo. Nhưng, hoặc là thiếu các trị đo, hoặc là thiếu thông tin về độ chính xác của điểm khống chế trắc địa. Nên bình sai lưới tự do trở thành đặc trưng của quá trình phân tích biến dạng. Bình sai lưới tự do, hay còn gọi là bình sai lưới tự do khuyết hạng [5] thường đề cập đến 5 phương pháp kinh điển sau:

- Phương pháp ma trận nghịch đảo tổng quát để giải hệ phương trình tuyến tính;

- Phương pháp trị đo giả, do Pelzer đề xuất năm 1974;

- Phương pháp thêm điều kiện phụ, do Mittermayer đề xuất năm 1972;

- Phương pháp giải trực tiếp, do Wolf đề xuất năm 1972;

- Phương pháp khử điều kiện, do Perelmuter đề xuất năm 1979 .

Tại Việt Nam, các kỹ sư trắc địa thường sử dụng phương pháp bình sai lưới tự do "thêm điều kiện ràng buộc nội" là ma trận C. Điều kiện này được tính toán nhích dần trên cơ sở hệ số $q \leq$ $t . m_{q_{c s}}$, với $\mathrm{t}$ là hệ số xác định tiêu chuẩn sai số giới hạn thường lấy trong khoảng $(2 \div 3), m_{q_{c s}}$ là yêu cầu về độ ổn định của điểm trắc địa. Ma trận $C_{i}=B_{i}$ đối với các điểm ổn định, ma trận $C_{i}=0$ đối với các điểm bất ổn định. Ma trận $B_{i}$ là ma trận chuyển đổi trong phép chuyển tọa độ Helmert và là tham số định vị lưới tự do. Quy trình phân tích độ ổn định của mạng lưới quan trắc biến dạng theo phương pháp lặp nhích dần sau:

Bước 1: Trong chu kỳ đang xét, thực hiện bình sai lưới tự do với một điểm Fix tọa độ (định vị tạm thời);

Bước 2: Tính độ lệch tọa độ của tất cả các điểm cơ sở so với tọa độ các điểm Fix ở chu kỳ đầu và tính chuyển tọa độ sau bình sai của các điểm trong mạng lưới về hệ tọa độ mới với điều kiện định vị mới;

Bước 3: Tính lại độ lệch tọa độ của các điểm cơ sở và áp dụng tiêu chuẩn $q \leq t . m_{q_{c S}}$ để kiểm tra và đánh giá độ ổn định của các điểm cơ sở trong lưới.

Bước 4: Kiểm tra, đánh giá độ ổn định các điểm cơ sở $\left(C_{i}=B_{i}\right)$ trong lưới. Có thể xảy ra một trong hai khả năng sau:

- Nếu phát hiện một hoặc một số mốc cơ sở không ổn định, thì sẽ loại điểm có độ lệch lớn nhất ra khỏi nhóm điểm ổn định bằng cách gán cho điểm đó giá trị $\left(C_{i}=0\right)$ và tính chuyển tọa độ theo điểm định vị mới;

- Nếu các điểm còn lại có $\left(C_{i}=B_{i}\right)$ thì kết thúc quá trình kiểm tra. Lưới được định vị gần đúng nhất so với điểm ổn định.

Quy trình này tồn tại một số vấn đề sau:

i. Tiêu chuẩn ban đầu đặt ra đối với quá trình bình sai lưới tự do là "trọng tâm của lưới không thay đổi trong quá trình xử lý bình sai". Dường như bước 2 của quy trình này đã vi phạm khi "tính chuyển tọa độ sau bình sai của các điểm trong mạng lưới về hệ tọa độ mới với điều kiện định vị mới.

ii. Việc áp dụng một tiêu chuẩn để nhận dạng điểm bất ổn định dường như thiếu chặt chẽ. Nếu có lưới độ cao 4 điểm, trong đó 3 điểm không ổn định được nhận dạng bằng tiêu chuẩn này. Vậy lưới đó có sử dụng được hay không?

iii. Đối với lưới quan trắc biến dạng. Tiêu chuẩn thống nhất về kết cấu lưới và đồ hình lưới trong các chu kỳ đo là rất quan trọng, nhằm giảm thiểu ảnh hưởng của sai số hệ thống đến kết quả xử lý lưới. Bước 4 của quy trình này đã vi phạm nghiêm trọng nguyên tắc ban đầu đã thống nhất.

iv. Xử lý lưới quan trắc biến dạng theo quy trình này là Fix điểm $i$, sau quá trình tính lặp nhích dần, để loại bỏ điểm không ổn định và định vị mạng lưới theo điểm i đã Fix. Nếu chu kỳ sau, chính bản thân điểm $\mathrm{i}$ đó cũng bị dịch chuyển. Quy trình tiếp tục Fix vào điểm $k$ khác. Như vậy, trọng tâm của lưới đã bị thay đổi và vi phạm điều 
kiện ban đầu đã thống nhất. Nếu cố tình sử dụng nó thì mạng lưới đang xét không thống nhất giữa các chu kỳ khác nhau và với chu kỳ đầu. Điều này vi phạm quy tắc bình sai lưới, dẫn đến kết quả chuyển dịch bị sai lệch, do không được so sánh với một gốc cố định.

Mục tiêu của nghiên cứu này là giải quyết các vấn đề đã nêu trên, đồng thời ứng dụng các thành tựu nghiên cứu của Ủy ban 6 về Phân tích biến dạng do Hội Các nhà Khảo sát quốc tế (FIG) đề xuất.

\section{2. Đối tượng và phương pháp nghiên cứu}

\subsection{Bình sai luới tụ do theo phưong pháp của Mittermayer}

Các điểm cơ sở trong lưới quan trắc biến dạng có thể cho là ổn định, cho đến khi phân tích thấy cấu trúc không ổn định của nó. Điều đó có nghĩa là, mạng lưới đó tự bản thân nó không mang đầy đủ các thông tin về độ chính xác trong không gian. Ví dụ lưới mặt bằng thiếu tọa độ điểm và phương vị mà chỉ có các liên kết giữa các điểm trong lưới. Do đó, một mạng lưới tự do là mạng lưới có thể chuyển dịch hoặc quay hoặc thu phóng tự do trong không gian của một hệ quy chiếu xác định. Đối với quá trình biến dạng của một vật thể, các nhà khoa học thế giới [5] đã thống nhất sử dụng biến đổi vi phân thay cho biến đổi Helmert để mô tả hệ tọa độ. Khi mạng lưới đó có một tọa độ và phương vị một cạnh (đối với lưới mặt bằng), lưới đó trở thành lưới tự do kinh điển, có số lượng gốc tối thiểu.

Quan tâm đến mô hình hàm số và mô hình ngẫu nhiên của mạng lưới tự do như sau [5]:

$$
\begin{array}{r}
l+v=A \hat{x}, \\
\sigma_{0}^{2} Q .
\end{array}
$$

ở đây $l$ là vector của $\mathrm{n}$ trị đo; $\mathrm{v}$ là vector số hiệu chỉnh của $\mathrm{n}$ trị đo; $\hat{x}$ là vector nghiệm (vector số hiệu chỉnh của tọa độ gần đúng của các điểm lưới); A là ma trận hệ số của cấu hình lưới; $\sigma_{0}^{2}$ là phương sai tiên nghiệm (phương sai trọng số đơn vị) và $Q$ ma trận đảo phương sai của trị đo (còn gọi là ma trận trọng số đảo). Đối với các trị đo độc lập, $Q$ là ma trận đường chéo nên không xuất hiện hiệp phương sai của các trị đo và cũng không có hiệp trọng số đảo của các trị đo. Nếu các trị đo là tương quan, như trong chuỗi trị đo GPS liên tục, sẽ tồn tại hiệp phương sai và hiệp trọng số đảo của trị đo. Đương nhiên, đối với ẩn số $\hat{x}$, sẽ tồn tại hiệp trọng số đảo của ẩn số. Tiếp theo ta có phương trình chuẩn dạng ma trận theo phương pháp số bình phương nhỏ nhất.

$$
N \hat{x}=w .
$$

ở đây $N=A^{T} Q^{-1} A, w=A^{T} Q^{-1} l$.

Do thiếu điều kiện gốc tối thiểu nên A khuyết hạng, dẫn tới ma trận hệ số $\mathrm{N}$ của phương trình chuẩn là suy biến. $\operatorname{det}\{N\}=0$. (phương trình chuẩn không có nghiệm duy nhất).

Bình sai lưới tự do khuyết hạng phải tuân thủ theo hai nguyên tắc:

$$
\begin{aligned}
& 1 / V^{T} P V=\min ; \\
& 2 /\|\hat{x}\|=\sqrt{\hat{x}^{T} \hat{x}}=\min . \text { Rút ra: } \hat{x}^{T} \hat{x}=\min .
\end{aligned}
$$

Điều kiện thứ hai nghĩa là, chuẩn của vector nghiệm phải nhỏ nhất.

Để giải bài toán bình sai lưới tự do theo phương pháp gián tiếp kèm điều kiện, ta cần phải định nghĩa điều kiện nội bộ để tìm ẩn số $\hat{x}$, biểu diễn bởi một hệ thống ràng buộc hay còn gọi là phương trình điều kiện như sau

$$
D^{T} \hat{x}=0 \text {, }
$$

Giả thiết $\hat{x}=\left(x_{1} x_{2} \ldots x_{n}\right)^{T}$ là một nghiệm thỏa mãn phương trình chuẩn, thì căn bậc hai của tổng bình phương của nó [4]:

$$
\|\hat{x}\|=\left(\hat{x}^{T} \hat{x}\right)^{\frac{1}{2}}=\frac{\sqrt{x_{1}^{2}+x_{2}^{2}+\cdots+x_{n}^{2}}}{n}
$$

gọi là chuẩn (norm hay module) của vector $\hat{x}, y$ nghĩa hình học là chiều dài (độ lớn) của vector. Nếu trong nghiệm chung của phương trình chuẩn có một nghiệm $\hat{x}$ thỏa mãn chuẩn nhỏ nhất, thì gọi nghiệm đó là nghiệm chuẩn nhỏ nhất, điều kiện thỏa mãn chuẩn nhỏ nhất gọi là điều kiện chuẩn nhỏ nhất, được biểu thị:

$$
\|\hat{x}\|=\min h o a ̣ ̆ c \hat{x}^{T} \hat{x}=\min
$$

Giả thiết $N_{m}^{-}$là một nghịch đảo tổng quát dạng $N^{-}$của $\mathrm{N}$, phương trình chuẩn có nghiệm riêng [6] là: 


$$
\hat{x}=N_{m}^{-} A^{T} P l
$$

Nếu chuẩn $N_{m}^{-} A^{T} P l$ của nghiệm riêng này nhỏ hơn chuẩn của bất kỳ nghiệm khác thì nó chính là nghiệm chuẩn nhỏ nhất. Vấn đề bây giờ là xác định $N_{m}^{-}$.

Theo Mittermayer,

$$
N_{m}^{-}=N^{T}\left(N N^{T}\right)^{-}
$$

Vì $\mathrm{N}$ là ma trận hệ số đối xứng. Do đó

$$
N_{m}^{-}=N(N N)^{-}
$$

Ta có nghiệm chuẩn nhỏ nhất của phương trình chuẩn

$$
\hat{x}=N(N N)^{-} A^{T} P l=N^{-1} A^{T} P l
$$

Do giả nghịch đảo $N^{+}$của $N$ cũng là một nghịch đảo chuẩn nhỏ nhất. Dùng $N^{+}=N_{m}^{-}=$ $N^{-1}=N(N N)^{-} N(N N)^{-} N .[6]$

Giải phương trình (2) với phương trình điều kiện $D^{T} \hat{x}=0$, ta có

$$
\hat{x}=\left(N+D D^{T}\right)^{-1} w,
$$

với ma trận hiệp trọng số đảo

$$
Q_{\hat{x}}=\left(N+D D^{T}\right)^{-1} H\left(H^{T} D D^{T} H\right)^{-1} H^{T},
$$

với ma trận $\mathrm{H}$ không suy biến với $\operatorname{rank}\{H\}=$ $\operatorname{rank}\{D\}$ và $\mathrm{NH}=0$.

Lời giải của phương trình (2) chú ý tới phương trình điều kiện $D^{T} \hat{x}$ có thể còn được thực hiện thông qua phép đổi cơ sở [5] từ bất kỳ lời giải $\hat{x}_{u}$ như sau

với

$$
\hat{x}=S \hat{x}_{u}, Q_{\hat{x}}=S Q_{\hat{x}_{u}} S^{T},
$$

$$
S=I-H\left(D^{T} H\right)^{-1} D^{T}=I-H\left(H^{T} W H\right)^{-1} H^{T} W,
$$

ở đây, $W=D\left(D^{T} D\right)^{-1} D^{T}$.

Ma trận $\mathrm{W}$ trong phương trình (12) còn được giải thích là ma trận trọng số khi định nghĩa điều kiện (3), và phương trình (11) còn được gọi là biến đồi tuần tư trọng số [7].

Nếu tất cả các điểm trong mạng lưới trong điều kiện (3) được định nghĩa là quan trọng như nhau, thì $W=I$ và ta có lời giải ràng buộc nội bộ. Nếu chỉ có một vài điểm được sử dụng để định nghĩa bài toán, những điểm đó nhận được trọng số đơn vị và những điểm khác nhận trọng số bằng 0 , ví $d u ̣, ~ W=\operatorname{diag}\{\mathrm{I}, 0\}$.
Phương sai hậu nghiệm $\hat{\sigma}_{0}^{2}$ và bậc tự do của nó, $d f$, được tính từ ước lượng số hiệu chỉnh $v$ như sau:

$$
\begin{gathered}
\hat{\sigma}_{0}^{2}=\frac{\hat{v}^{T} Q^{-1} \hat{v}}{d f}, \\
d f=n-\operatorname{rank}\{A\},
\end{gathered}
$$

ở đây, bậc của $\mathrm{A}$ đối với lưới có cấu hình đầy đủ (không khuyết) là đủ số lượng cho các tham số là ẩn số còn thiếu trong điều kiện khuyết (3) của lưới [8].

\subsection{Cực tiểu hóa chuẩn bậc nhất}

Khi so sánh 2 chu kỳ đo, vector dịch chuyển của tất cả các điểm quan trắc và ma trận phương sai của nó được tính:

$$
d=\hat{x}_{2}-\hat{x}_{1}, \quad Q_{d}=Q_{\hat{x}_{2}}+Q_{\hat{x}_{1}},
$$

Yếu tố phương sai chung $\hat{\sigma}_{0_{p}}^{2}$ và bậc tự do của nó $d f_{p}$ được tính [8]:

$$
\begin{gathered}
\hat{\sigma}_{0_{p}}^{2}=\frac{\left[d f_{1}\left(\hat{\sigma}_{0_{01}}^{2}\right)+d f_{2}\left(\hat{\sigma}_{0_{02}}^{2}\right)\right]}{d f_{p}}, \\
d f_{p}=d f_{1}+d f_{2},
\end{gathered}
$$

ở đây, số dưới 1 và 2 để chỉ chu kỳ 1 và 2 . Nếu phương sai tiên nghiệm không thông qua được kiểm định thống kê với giả thiết $H_{0}: \hat{\sigma}_{0_{01}}^{2}=\hat{\sigma}_{0_{02}}^{2}$, với mức ý nghĩa thống kê $\alpha$

$$
\begin{aligned}
& {\left[F\left(\alpha / 2, d f_{2}, d f_{1}\right)\right]^{-1}<\frac{\left(\hat{\sigma}_{0_{01}}^{2}\right)}{\left(\hat{\sigma}_{0_{02}}^{2}\right)}} \\
& <F\left(\alpha / 2, d f_{2}, d f_{1}\right),
\end{aligned}
$$

nghĩa là có lỗi trong kiểm định trên, nguyên nhân là trọng số so sánh của trị đo giữa 2 chu kỳ hoặc trọng số của đồ hình lưới không chính xác (đồ hình lưới quan trắc hai chu kỳ khác nhau).

Như đã đề cập, tính toán dịch chuyển bằng phương trình (14) có thể không chính xác bởi điều kiện ràng buộc nội đã lựa chọn hoặc phải định nghĩa điều kiện ràng buộc nội khác trong quá trình bình sai 2 chu kỳ, do đó làm cho việc xác định điểm cơ sở không ổn định thêm khó khăn. Để giải quyết vấn đề này, cần cực tiểu hóa chuẩn bậc nhất của vector dịch chuyển của điểm 
cơ sở [9]. Chiến lược ấy cung cấp điều kiện vững cho việc kiểm định điểm cơ sở không ổn định và nhận được vector dịch chuyển ít bị sai sót nhất [7].

Bắt đầu với $d_{\tau}$ và $Q_{d_{\tau}}$ là vector dịch chuyển và ma trận phương sai của điểm cơ sở. Từ $d$ và $Q_{d}$ trong phương trình (14). Ta biến đổi chúng tới phương trình điều kiện khác phù hợp hơn với phương trình (11), (12) như sau:

$$
\tilde{d}_{\tau} \simeq\left[I-H_{\tau}\left(H_{\tau}^{T} W_{\tau} H_{\tau}\right)^{-1} H_{\tau}^{T} W_{\tau}\right] d_{\tau} \simeq S_{\tau} d_{\tau}
$$
và

$$
Q_{\tilde{d}_{\tau}} \simeq S_{\tau} Q_{d_{\tau}} S_{\tau}^{T}
$$

Ma trận $H_{\tau}$ có cấu trúc như trước và phụ thuộc vào phương trình điều kiện ràng buộc nội đặt ra ban đầu trong 2 chu kỳ đối với các điểm cơ sở. Ví dụ, nếu lưới kiểm tra trong chu kỳ đầu là lưới tam giác đo góc cạnh có phương trình điều kiện ràng buộc nội, xác nhận trong chu kỳ thứ hai lưới bị dịch chuyển, quay, thu phóng. Phương trình điều kiện ràng buộc nội của lưới sẽ xác nhận hai chiều dịch chuyển $(\mathrm{x}, \mathrm{y})$ và một chiều quay. Sau đó, liên kết điều kiện ràng buộc nội như là trong chu kỳ đầu.

Chiến lược hiện nay là, lựa chọn ma trận trọng số $W_{\tau}$ trong phương trình (17a) làm chuẩn bậc nhất của vector dịch chuyển $\tilde{d}_{\tau}$ xấp xỉ cực tiểu. Ví dụ, $\left\|\tilde{d}_{\tau}\right\|_{i}=\min$, nghĩa là chuẩn của vector $\tilde{d}_{\tau}$ trong không gian Euclide được tối thiểu hóa. Đặt

$$
t=\left(H_{\tau}^{T} W_{\tau} H_{\tau}\right)^{-1} H_{\tau}^{T} W_{\tau} d_{\tau},
$$

được gọi là tham số chuyển đổi. Sau đó đặt

$$
\left\|\tilde{d}_{\tau}\right\|_{i}=\sum_{i}\left|d_{\tau}(i)-h_{i} t\right|,
$$

ở đây, $d_{\tau}(i)$ là phân tử thứ i của $d_{\tau}$ và $h_{i}$ là vector hàng thứ i của ma trận $H_{\tau}$. Điều kiện này có thể viết như sau:

$$
\min _{t} \sum_{i}\left|d_{\tau}(i)-h_{i} t\right|
$$

Phương trình (18) không phải luôn giải được. Tuy nhiên, đối với việc xác nhận điểm cơ sở không ổn định thì không thành vấn đề.
Đối với lưới kiểm tra độ cao (đo lún), các tham số của phương trình điều kiện ràng buộc nội có lượng chuyển dịch $t_{z}$ theo chiều dây dọi. Nếu $w_{i}$ là dịch chuyển của điểm $P_{i}$ thì từ (18) ta có

$$
\min _{t z} \sum_{i}\left|w_{i}-t_{z}\right|,
$$

Lời giải đối với $t_{z}$ là rõ ràng. Tất cả $w_{i}$ được sắp xếp lại (chỉnh hợp) vào một chuỗi các giá trị đại số tăng dần của chúng, và giá trị trung bình là giá trị $t_{z}$. Nếu đó là số tương đương nhau của điểm cơ sở, thì giá trị khác của 2 chuyển dịch ở giữa hoặc trung bình của chúng có thể được sử dụng như là $t_{z}$. Nói cách khác, điểm hoặc cặp điểm trong chuyển dịch thì thuộc về vùng giữa có trọng số bằng 1 , và những điểm còn lại thì có trọng số bằng 0 . Vector mới của dịch chuyển và ma trận hiệp trọng số của nó được tính từ phương trình (17).

Đối với lưới hai chiều, phương pháp Tuần tự biến đổi trọng số phức tạp hơn nhiều. Trong phương pháp này, ma trận trọng số $W_{\tau}$ trong phương trình (17) sẽ được xem như khởi đầu, sau đó, tại lần biến đổi thứ $(\mathrm{k}+1)$, ma trận trọng số được xác định như sau:

$$
W_{\tau}^{(k+1)}=\operatorname{diag} \frac{1}{\left|\tilde{d}_{\tau}^{k}(i)\right|},
$$

ở đây $\tilde{d}_{\tau}^{k}(i)$, là thành phần thứ $\mathrm{i}$ của vector $\tilde{d}_{\tau}$ sau lần tính thứ k. Quá trình tính lặp tiếp tục cho tới khi sự khác nhau thuần túy giữa những lần biến đổi kế tiếp thành phần chuyển dịch mặt bằng nhỏ hơn một dung sai $\delta$ (khoảng $1 / 2$ độ chính xác trung bình của thành phần chuyển dịch mặt bằng). Trong suốt quá trình này, một vài $\tilde{d}_{\tau}^{k}(i)$ có thể xấp xỉ bằng 0 , nguyên nhân do quá trình làm tròn số, bởi vì thành phần $\frac{1}{\left|\tilde{d}_{\tau}^{k}(i)\right|}$ rất lớn. Có hai cách giải quyết vấn đề này. Cách thứ nhất, thay đổi biểu thức thứ (20) bằng:

$$
W_{\tau}^{(k+1)}=\operatorname{diag} \frac{1}{\left|\tilde{d}_{\tau}^{k}(i)\right|+\delta}
$$

Cách thứ hai là đặt một cận dưới. Khi $\left|\tilde{d}_{\tau}^{k}(i)\right|$ nhỏ hơn cận dưới đó thì trọng số của nó tiến tới 0 . Nếu trong lần lặp tiếp theo, $\left|\tilde{d}_{\tau}^{k}(i)\right|$ lớn hơn thì 
trọng số có thể được thay đổi theo cho phù hợp hơn. [7].

Cả hai quy trình trên cung cấp lời giải xấp xỉ cho phương trình (18). Trong lần lặp cuối cùng, lần thứ $(\mathrm{k}+1)$, ma trận trọng số đảo được tính như sau:

$$
Q_{\tilde{d}_{\tau}}=S_{\tau}^{(k+1)} Q_{d_{\tau}}\left[S_{\tau}^{(k+1)}\right]^{T} .
$$

Bằng việc so sánh dịch chuyển của mỗi điểm dựa vào vùng tin cậy của chúng tại mức ý nghĩa thống kê $\alpha$, ta có thể thấy rằng các điểm cơ sở hầu hết không ổn định.

\section{3. Ước lương điểm không ổn định}

Các điểm cơ sở được xác định là không ổn định và tất cả các điểm kiểm tra sẽ cùng được đưa vào bình sai theo phương pháp bình phương nhỏ nhất của mô hình biến dạng $\mathrm{Bc}$ với những giá trị dịch chuyển $\mathrm{d}$ nhận được theo phương trình [10]:

$$
d+v=B c,
$$

ở đây, $v$ là vector của độ lệch sau khi hiệu chỉnh (số hiệu chỉnh cho trị đo), c là vector dịch chuyển cuối cùng đã được ước lượng và $B$ là ma trận hệ số cấu hình lưới.

Rõ ràng, mô hình biến dạng của mỗi điểm cơ sở không ổn định và điểm kiểm tra $P_{i}$ trong lưới hai chiều được viết:

$$
d_{i}+v_{i}=\left[\begin{array}{l}
a_{i} \\
b_{i}
\end{array}\right]=c_{i},
$$

và đối với điểm ổn định $P_{j}$ như sau:

$$
d_{j}+v_{j}=\left[\begin{array}{l}
0 \\
0
\end{array}\right] \text {, }
$$

Do đó, ma trận $B$ trong phương trình (22) có các phần tử dạng đơn vị tương ứng với những điểm không ổn định và những điểm kiểm tra, còn những điểm khác thì bằng 0 . Lời giải của phương trình (22) như sau:

$$
\hat{c}=\left(B^{T} P_{d} B\right)^{-1} B^{T} P_{d} d,
$$

và ma trận trọng số đảo của nó là:

$$
Q_{\hat{c}}=\left(B^{T} P_{d} B\right)^{-1} \text {. }
$$

Ma trận trọng số được tính theo một cách khác là:

$$
\begin{array}{cc} 
& P_{d}=N_{1}\left(N_{1}+N_{2}\right)^{-} N_{2} . \\
\text { hoặc } & N_{1}=\left(S Q_{d} S\right)^{+}=\left[S Q_{d} S+\right. \\
\left.H\left(H^{T} H\right)^{-1} H^{T}\right]^{-1}-H\left(H^{T} H\right)^{-1} H^{T} .
\end{array}
$$

trong phương trình (25) có $N_{1}, N_{2}$ là ma trận hệ số của phương trình chuẩn (3). Nghịch đảo tổng quát $\left(N_{1}+N_{2}\right)^{-}$ta có thể tính được $\left(N_{1}+N_{2}+\right.$ $\left.H H^{T}\right)^{-1}[1,2]$. Ở đây, vector cột $\mathrm{H}$ tương ứng với số khuyết của lưới tự do tổng quát trong hai chu kỳ. Nếu hai chu kỳ có chung một cấu hình lưới và các trị đo có độ chính xác tương đương, ta có: $N_{1}=N_{2}=N$ và:

$$
P_{d}=N / 2 \text {. }
$$

Trong phương trình (26) ma trận $\mathrm{S}$ được biểu diễn trong phương trình (24) với $W=I$ và vector cột $\mathrm{H}$ tương ứng với số khuyết của lưới tự do hợp nhất trong hai chu kỳ. Lý do phải tính ma trận trọng số theo cách như vậy là để ước lượng ẩn số $\hat{c}$ sao cho độc lập trong điều kiện ràng buộc nội của bài toán bình sai. Nếu điều kiện khuyết bị khử bởi trị đo giả có phương sai nhỏ đã giới thiệu ban đầu, thì ma trận trọng số được tính:

$$
P_{d}=Q_{d}^{-1} \text {. }
$$

Tuy nhiên, trong trường hợp này, không chỉ có vấn đề về số học có khả năng xảy ra do điều kiện $Q_{d}$ xấu, mà còn xảy ra sự phức tạp phát sinh trong mô hình biến dạng [8].

Ý nghĩa của việc ước lượng dịch chuyển $\hat{c}_{i}$ đối với điểm cơ sở không ổn định $P_{i}$ được thực hiện bằng kiểm định:

$$
\frac{\hat{c}_{i}^{T} Q_{c_{i}}^{-1} \hat{c}_{i}}{m_{c} \widehat{\sigma}_{0_{P}}^{2}}>F\left(\alpha, m_{c}, d f_{P}\right) \text {. }
$$

ở đây, $m_{c}$ chính là thứ hạng của $\hat{c}_{i} ; Q_{\hat{c}_{i}}$ chính là ma trận con của $Q_{\hat{c}}$ và $\hat{\sigma}_{0_{P}}^{2}$ và $d f_{P}$ là thành phần phương sai gộp chung và bậc tự do của nó.

Để kiểm định giả thiết rằng không còn điểm bất ổn định nào tồn tại nữa, hàm bậc hai $\Delta R$ của ước lượng độ lệch $\hat{v}$ được tính như sau:

$$
\Delta R=\hat{v}^{T} P_{d} \widehat{v} .
$$

Kiểm định Khi bình phương với bậc tự do như sau: 


$$
d f_{c}=(\operatorname{dim}(d)-1)-m_{c}
$$

ở đây, $m_{c}$ là thứ hạng của vector $\mathrm{c}$ chưa biết; $(\operatorname{dim}(d)-1)=d f_{s}$ là số lượng các chênh cao độc lập. Nếu bất đăng thức

$$
\frac{\Delta R}{d f_{c} \widehat{\sigma}_{0_{P}}^{2}}<F\left(\alpha, d f_{c}, d f_{P}\right) \text {. }
$$

tồn tại, thì giả thiết được chấp nhận tại mức ý nghĩa $(1-\alpha) \%$.

Nói cách khác, khảo sát đối với điểm cơ sở không ổn định khác cũng nên được làm. Khi yếu tố phương sai tiên nghiệm $\sigma_{0}^{2}$ đã biết, thành phần $\hat{\sigma}_{0_{P}}^{2}$ và $d f_{P}$ trong kiểm định $(30)$ và $(27)$ được thay thế bởi $\sigma_{0}^{2}$ và $\infty$ theo thứ tự lần lượt.

\subsection{Luới mặt bằng thủy điện Tuyên Quang}

Có 7 điểm cơ sở trong hệ thống mốc khống chế xây dựng cơ bản của nhà máy. Nhưng chỉ lấy 4 mốc có kết cấu trên nền đá gốc, vị trí thuận tiện cho quan trắc biến dạng, tạo thành kết cấu đồ hình vững cho lưới quan trắc biến dạng. Đó là các mốc: QT01, QT03, QT05 và QT06. Sử dụng máy toàn đạc điện tử Leica độ chính xác đo cạnh $(1+1 \mathrm{ppm}) \mathrm{mm}$, đo góc toàn vòng và đo cạnh theo hai chiều đi - về, tham khảo thêm trị đo GPS theo kỹ thuật Tương đối-Tĩnh. Chu kỳ đo tháng giêng năm 2013, chu kỳ đo tháng 6 năm 2013, chu kỳ đo tháng giêng năm 2014. Hình 1 là lưới cơ sở mặt bằng thủy điện Tuyên Quang với ba chu kỳ đo. Các giá trị quan trắc [11] ở bảng 1 :

Bảng 1

\begin{tabular}{lllll}
\hline $\begin{array}{l}\text { Tuyến } \\
\text { đo }\end{array}$ & Tên cạnh & $\begin{array}{l}\text { Chiều dài }(\mathrm{m}) \\
- \text { Chu kỳ } \\
(01 / 2013)\end{array}$ & $\begin{array}{l}\text { Chiều dài }(\mathrm{m}) \\
- \text { Chu kỳ } \\
(6 / 2013)\end{array}$ & $\begin{array}{l}\text { Chiều dài }(\mathrm{m}) \\
- \text { Chu kỳ 3 } \\
(01 / 2014)\end{array}$ \\
\hline L1 & QT3-QT6 & 956.716 & 956.714 & 956.712 \\
L2 & QT1-QT6 & 1191.106 & 1191.111 & 1191.108 \\
L3 & QT5-QT6 & 464.597 & 464.598 & 464.595 \\
L4 & QT3-QT5 & 1218.583 & 1218.581 & 1218.577 \\
L5 & QT1-QT3 & 610.630 & 610.632 & 610.629 \\
L6 & QT1-QT5 & 1223.244 & 1223.245 & 1223.242 \\
\hline
\end{tabular}

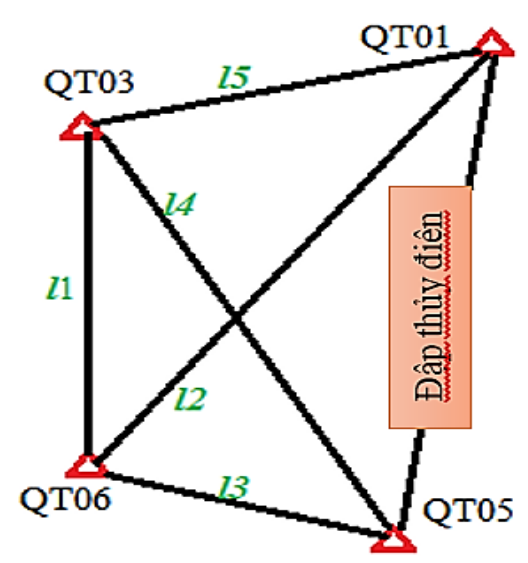

Hình 1. Lưới cơ sở mặt bằng thủy điện Tuyên Quang. 
Bước 1. Bình sai lưới không ràng buộc và tính sự dịch chuyển.

Giả định tọa độ gần đúng của điểm QT06 là $\mathrm{X}_{\mathrm{QT} 06}^{0}=0, \mathrm{Y}_{\mathrm{QT} 06}^{0}=0$, phương vị cạnh QT06 QT03 là $\alpha_{\mathrm{QT06-QT03}}^{0}=0^{0}$. Tức giả định điểm gốc của lưới là QT06, trục $\mathrm{OX}$ đi từ QT06 đến QT03, do vậy giá trị $Y_{Q T 3}=0 \mathrm{~mm}$ và giá trị $X_{Q T 3}$ đúng bằng chiều dài cạnh QT3-QT6 đo được. Từ đó tính tọa độ gần đúng các điểm còn lại.

Chu kỳ 1 .

Bảng 2. Ma trận N.N:

\begin{tabular}{llllllll}
\hline 5.510 & 0.293 & -3.472 & 0.635 & -2.911 & -1.796 & 0.873 & 0.868 \\
0.293 & 2.319 & -0.635 & 0.528 & -1.711 & -0.987 & 2.053 & -1.860 \\
-3.472 & -0.635 & 5.510 & -0.867 & 1.453 & 0.414 & -3.490 & 1.088 \\
0.635 & 0.528 & -0.867 & 2.389 & -1.302 & -2.447 & 1.534 & -0.469 \\
-2.911 & -1.711 & 1.453 & -1.302 & 5.212 & 2.782 & -3.754 & 0.231 \\
-1.796 & -0.987 & 0.414 & -2.447 & 2.782 & 3.362 & -1.399 & 0.072 \\
0.873 & 2.053 & -3.490 & 1.534 & -3.754 & -1.399 & 6.370 & -2.187 \\
0.868 & -1.860 & 1.088 & -0.469 & 0.231 & 0.072 & -2.187 & 2.256 \\
\hline
\end{tabular}

Chu kỳ 1 .

Bảng 3. Nghịch đảo chuẩn nhỏ nhất của $N_{m}^{-}$

\begin{tabular}{llllllll}
\hline 0.445 & 0.081 & 0.074 & -0.056 & 0.099 & 0.000 & 0.000 & 0.000 \\
0.130 & 0.606 & 0.106 & -0.051 & 0.146 & 0.000 & 0.000 & 0.000 \\
-0.001 & 0.032 & 0.370 & -0.018 & -0.100 & 0.000 & 0.000 & 0.000 \\
-0.004 & -0.051 & 0.012 & 0.530 & 0.089 & 0.000 & 0.000 & 0.000 \\
0.028 & 0.071 & -0.067 & 0.137 & 0.424 & 0.000 & 0.000 & 0.000 \\
-0.169 & -0.074 & -0.194 & -0.446 & -0.042 & 0.000 & 0.000 & 0.000 \\
-0.472 & -0.183 & -0.377 & -0.064 & -0.423 & 0.000 & 0.000 & 0.000 \\
0.042 & -0.482 & 0.076 & -0.033 & -0.192 & 0.000 & 0.000 & 0.000 \\
\hline
\end{tabular}

Chu kỳ 1.

Bảng 4. Nghiệm xác suất nhất

\begin{tabular}{lllll}
\hline Điểm cơ sở & QT6 & QT3 & QT1 & QT5 \\
\hline $\mathrm{X}^{\prime}(\mathrm{m})$ & 0.0005 & -0.0005 & 0.0023 & -0.0023 \\
$\mathrm{Y}^{\prime}(\mathrm{m})$ & 0.0008 & 0.0005 & -0.0002 & -0.0011 \\
\hline
\end{tabular}

Chu kỳ 1.

Bảng 5. Tọa độ cuối cùng tính được

\begin{tabular}{lllll}
\hline Điểm cơ sở & QT6 & QT3 & QT1 & QT5 \\
\hline $\mathrm{X}(\mathrm{m})$ & 0.001 & 956.715 & 1024.951 & -184.900 \\
$\mathrm{Y}(\mathrm{m})$ & 0.001 & 0.000 & 606.806 & 426.219 \\
\hline
\end{tabular}


Tương tự như cách tính của chu kỳ 1 khi giả định điểm gốc và phương vị gốc của lưới, các chu kỳ thứ 2 và 3 thì tọa độ gần đúng (số liệu đầu vào) được xác định căn cứ vào kết quả bình sai chu kỳ trước (bảng 5 đối với chu kỳ 2 và bảng 6 đối với chu kỳ 3). Nghĩa là, trọng tâm lưới luôn luôn không thay đổi. Ta thấy, hầu hết các điểm trong lưới không ổn định theo kết quả quan trắc các chu kỳ. Tọa độ cuối cùng như sau:

Chu kỳ 2.

Bảng 6. Tọa độ cuối cùng tính được

\begin{tabular}{ccccc}
\hline Điểm cơ sở & QT6 & QT3 & QT1 & QT5 \\
\hline X $(\mathrm{m})$ & 0.001 & 956.713 & 1024.958 & -184.901 \\
Y $(\mathrm{m})$ & 0.001 & 0.001 & 606.807 & 426.219 \\
\hline
\end{tabular}

Chu kỳ 3.

Bảng 7. Tọa độ cuối cùng tính được

\begin{tabular}{lllll}
\hline Điểm cơ sở & QT6 & QT3 & QT1 & QT5 \\
\hline X $(\mathrm{m})$ & 0.001 & 956.711 & 1024.956 & -184.899 \\
$\mathrm{Y}(\mathrm{m})$ & 0.001 & 0.001 & 606.804 & 426.217 \\
\hline
\end{tabular}

Bảng 8 . Tổng hợp kết quả 3 chu kỳ và tính độ lệch (m)

\begin{tabular}{lllll|lllll}
\hline Chu kỳ (2-1) & QT6 & QT3 & QT1 & QT5 & Chu kỳ (3-1) & QT6 & QT3 & QT1 & QT5 \\
\hline$x 2-x l=d_{x}^{1}$ & 0.0000 & 0.0027 & -0.0062 & 0.0011 & $x 3-x l=d_{x}^{2}$ & 0.0000 & 0.0047 & -0.0045 & -0.0001 \\
$y 2-y l=d_{y}^{1}$ & 0.0000 & 0.0002 & -0.0006 & -0.0002 & $y 3-y l=d_{y}^{2}$ & 0.0000 & 0.0002 & 0.0025 & 0.0025 \\
\hline
\end{tabular}

Thuật toán của Mittermayer chính là đưa điểm gốc trong lưới trắc địa cơ sở về trọng tâm của mạng lưới đó, thỏa mãn (4). Ta thấy rằng, hầu hết các điểm trong lưới không ổn định, lớn nhất là $\quad d_{x}^{1}(\max )=6,2 \mathrm{~mm} ; d_{x}^{2}(\max )=$ $4,7 \mathrm{~mm}$.
Buớc 2. Sử dụng thuật toán Biến đổi trọng số, xác định điểm chuyển dịch bằng cực tiểu hóa chuẩn bậc nhất vector chuyển dịch. Áp dụng công thức từ (14) đến (30). Kết quả tính ma trận W lần đầu chưa đạt điều kiện cực tiểu hóa.

Bảng 9. Kết quả tính lặp ma trận $\mathrm{W}$ vòng thứ 2 với điều kiện cận dưới $\pm 0,1 \mathrm{~mm}$ (tương đương sai số của máy đo)

\begin{tabular}{llllllll}
\hline 10000.0000 & 0.0000 & 0.0000 & 0.0000 & 0.0000 & 0.0000 & 0.0000 & 0.0000 \\
0.0000 & 10000.0000 & 0.0000 & 0.0000 & 0.0000 & 0.0000 & 0.0000 & 0.0000 \\
0.0000 & 0.0000 & 10000.0000 & 0.0000 & 0.0000 & 0.0000 & 0.0000 & 0.0000 \\
0.0000 & 0.0000 & 0.0000 & 10000.0000 & 0.0000 & 0.0000 & 0.0000 & 0.0000 \\
0.0000 & 0.0000 & 0.0000 & 0.0000 & 0.4238 & 0.0000 & 0.0000 & 0.0000 \\
0.0000 & 0.0000 & 0.0000 & 0.0000 & 0.0000 & 0.2834 & 0.0000 & 0.0000 \\
0.0000 & 0.0000 & 0.0000 & 0.0000 & 0.0000 & 0.0000 & 0.4225 & 0.0000 \\
0.0000 & 0.0000 & 0.0000 & 0.0000 & 0.0000 & 0.0000 & 0.0000 & 1.2867 \\
\hline
\end{tabular}

Ta có $\tilde{d}_{\tau}$ của lần tính lặp thứ hai.

Bảng 10. Giá trị $\tilde{d}_{\tau}(\mathrm{m})$

\begin{tabular}{cccccccc}
\hline$d x_{Q T 6}$ & $d y_{Q T 6}$ & $d x_{Q T 3}$ & $d y_{Q T 3}$ & $d x_{Q T 1}$ & $d y_{Q T 1}$ & $d x_{Q T 5}$ & $d y_{Q T 5}$ \\
\hline 0.0000 & 0.0000 & 0.0000 & 0.0000 & -0.0014 & -0.0001 & 0.0014 & 0.0006 \\
\hline
\end{tabular}


Bảng 11. Kết quả tính $P_{d}$

\begin{tabular}{llllllll}
\hline 0.1424 & 0.0427 & -0.0434 & -0.0370 & -0.0237 & -0.0100 & -0.0753 & 0.0043 \\
0.0427 & 0.1899 & 0.0303 & -0.0454 & -0.0089 & -0.0133 & -0.0641 & -0.1313 \\
-0.0434 & 0.0303 & 0.1419 & -0.0290 & -0.0710 & -0.0225 & -0.0275 & 0.0212 \\
-0.0370 & -0.0454 & -0.0290 & 0.1598 & 0.0633 & -0.1112 & 0.0028 & -0.0033 \\
-0.0237 & -0.0089 & -0.0710 & 0.0633 & 0.1497 & -0.0026 & -0.0549 & -0.0517 \\
-0.0100 & -0.0133 & -0.0225 & -0.1112 & -0.0026 & 0.1180 & 0.0352 & 0.0065 \\
-0.0753 & -0.0641 & -0.0275 & 0.0028 & -0.0549 & 0.0352 & 0.1577 & 0.0262 \\
0.0043 & -0.1313 & 0.0212 & -0.0033 & -0.0517 & 0.0065 & 0.0262 & 0.1281 \\
\hline
\end{tabular}

Sau đó xác định điểm có chuyển dịch nhiều nhất trong ma trận định vị lưới $\hat{c}=$ tính toán kiểm định Fisher theo công thức (27) $\left(B^{T} P_{d} B\right)^{-1} B^{T} P_{d} d$, bắt đầu với $B^{T}$. Quá trình và (30) đều đạt yêu cầu.

Bảng 12. Tìm $B^{T}$ đối với điểm QT5 và QT1

\begin{tabular}{llllllllllllllllll}
\hline \multirow{2}{*}{ QT5 } & 1 & 0 & 1 & 0 & 1 & 0 & 0 & 0 & \multirow{2}{*}{ QT1 } & 1 & 0 & 1 & 0 & 0 & 0 & 1 & 0 \\
& 0 & 1 & 0 & 1 & 0 & 1 & 0 & 0 & & 0 & 1 & 0 & 1 & 0 & 0 & 0 & 1 \\
\hline
\end{tabular}

Tìm được dịch chuyển của điểm QT5 thông qua $\hat{c}$

$$
\begin{array}{lllll}
\hline \text { QT5 } & \hat{c}_{x} & -0.0018(\mathrm{~m}) & \hat{c}_{y} & -0.0011(\mathrm{~m}) \\
\hline
\end{array}
$$

Tương tự, ta tìm được dịch chuyển của điểm QT1 thông qua $\hat{c}$

$$
\begin{array}{lllll}
\hline \text { QT1 } & \hat{c}_{x} & 0.0021(\mathrm{~m}) & \hat{c}_{y} & -0.0003(\mathrm{~m}) \\
\hline
\end{array}
$$

Vì khoảng dịch chuyển là cạnh huyền của tam giác vuông với các cạnh $\hat{c}_{x}$ và $\hat{c}_{y}$.

Bảng 13. Thành quả thực hiện Biến đổi tuần tự trọng số sau chu kỳ 2

\begin{tabular}{clll|llll}
\hline Trước khi thực hiện Biến đổi tuần tự trọng số & \multicolumn{4}{|c}{ Sau khi thực hiện Biến đổi tuần tự trọng số } \\
\hline$d_{Q T 6}(\mathrm{~m})$ & $d_{Q T 3}(\mathrm{~m})$ & $d_{Q T 1}(\mathrm{~m})$ & $d_{Q T 5}(\mathrm{~m})$ & $d_{Q T 6}(\mathrm{~m})$ & $d_{Q T 3}(\mathrm{~m})$ & $d_{Q T 1}(\mathrm{~m})$ & $d_{Q T 5}(\mathrm{~m})$ \\
\hline 0.0000 & 0.0027 & 0.0062 & 0.0011 & 0.0000 & 0.0000 & 0.0022 & 0.0021 \\
\hline
\end{tabular}

Bảng 14. Thành quả thực hiện Biến đổi trọng số sau chu kỳ 3

\begin{tabular}{llll|lllll}
\hline Trước khi thực hiện Biến đổi tuần tự trọng số & \multicolumn{4}{|c}{ Sau khi thực hiện Biến đổi tuần tự trọng số } \\
\hline$d_{Q T 6}(\mathrm{~m})$ & $d_{Q T 3}(\mathrm{~m})$ & $d_{Q T 1}(\mathrm{~m})$ & $d_{Q T 5}(\mathrm{~m})$ & $d_{Q T 6}(\mathrm{~m})$ & $d_{Q T 3}(\mathrm{~m})$ & $d_{Q T 1}(\mathrm{~m})$ & $d_{Q T 5}(\mathrm{~m})$ \\
0.0000 & 0.0047 & -0.0045 & -0.0001 & 0.0000 & 0.0000 & 0.0025 & 0.0027 \\
\hline
\end{tabular}

Đây thật sự là khác biệt. Dịch chuyển từ 2,2 $\mathrm{mm}$ đến $2,7 \mathrm{~mm}$ là xấp xỉ sai số đo lưới mặt bằng, khi giả thiết rằng: $\pm 1 \mathrm{~mm}$ là sai số do máy đo và từ $\pm 1 \mathrm{~mm}$ đến $\pm 1,5 \mathrm{~mm}$ là sai số do người đo trên mạng lưới cơ sở trắc địa thủy điện Tuyên Quang. Có thể thấy rằng, lưới trắc địa cơ sở đập thủy điện Tuyên Quang được xây dựng trên nền đá gốc khá ổn định. Mọi dịch chuyển dường như chỉ là sai số của kỹ thuật đo mà thôi.

\section{Thảo luận}

1.1. Sự khác biệt cơ bản của phương pháp Mittermayer so với phương pháp đang sử dụng bởi các kỹ sư trắc địa là sử dụng phương pháp "Giả nghịch đảo" để giải phương trình chuẩn khuyết hạng. Nó cho thấy sự giản đơn, tránh nhầm lẫn trong quá trình tính toán hoặc lập phần mềm. Như đã phân tích, tính toán bình sai lưới 
tự do bằng thêm "điều kiện ràng buộc nội" thường phải định nghĩa lại điều kiện ràng buộc, khiến cho việc xác định trung tâm lưới thêm khó khăn. Chiến lược ở đây là cực tiểu hóa chuẩn bậc nhất của vector tham số bình sai, điều này tương đồng với điều kiện $\|\hat{x}\|=\sqrt{\hat{x}^{T} \hat{x}}=\min$. Chiến lược ấy cung cấp điều kiện "vững" cho bài toán bình sai [12]. Đây là ưu điểm của phương pháp bình sai Mittermayer.

Nếu chỉ sử dụng bình sai lưới tự do và đặt một giá trị $q \approx \pm 1 \mathrm{~mm}$ để xác định chuyển dịch (cách làm truyền thống), điểm QT1 đạt giá trị max $6,2 \mathrm{~mm}$, QT3 đạt giá trị $2,7 \mathrm{~mm}$ (bảng 8 ) và đương nhiên bị coi là dịch chuyển, đồng thời bị loại ra khỏi lưới. Nếu sử dụng phương pháp "Biến đổi tuần tự trọng số", điểm QT1 giá trị dịch chuyển là $2,2 \mathrm{~mm}$ và QT5 là $2,1 \mathrm{~mm}$. Giá trị chuyển dịch đều thấp hơn so với cách làm truyền thống, đồng thời vị trí điểm chuyển dịch thay đổi từ QT3 sang QT5. Nhưng điều quan trọng là trọng tâm lưới không thay đổi. Vì cách làm truyền thống đã loại bỏ điểm chuyển dịch làm cho lưới chỉ còn hai điểm và trọng tâm lưới đã thay đổi. Đây là ưu điểm của phương pháp "Biến đổi tuần tự trọng số".

1.2. Nếu quan trắc nhiều chu kỳ, lưới có 4 điểm cơ sở trắc địa, chu kỳ đầu có thể điểm QT6 ổn định, nhưng chu kỳ sau không chắc điểm QT6 sẽ ổn định. Như vậy sẽ phải thay đổi điểm định vị lưới sau mỗi chu kỳ, làm cho kết cấu lưới không đồng nhất, khó có thể làm căn cứ để đánh giá các điểm mục tiêu quan trắc biến dạng công trình. Hơn nữa, từ những vị trí quan trắc khác nhau, ta sẽ nhận thấy đối tượng dịch chuyển theo phương khác nhau và giá trị chuyển dịch cũng khác nhau.

Chúng ta có một chiến lược để xác định trung tâm ổn định của lưới, dù cho lưới có bị chuyển dịch và hầu hết các điểm đều không ổn định (theo quy luật ngẫu nhiên, các điểm không cùng chuyển dịch về một hướng). Đó chính là "cực tiểu hóa chuẩn bậc nhất của vector trị đo" trong quá trình bình sai lưới. Đây cũng đồng thời là điều kiện "vững" theo lý thuyết thống kê "Robust Statistic" mà Peter Huber đề xuất [12].

1.3. Xác định điểm chuyển dịch và tính lượng chuyển dịch. Quá trình tính lặp ma trận W nhằm tìm ra "chuẩn bậc nhất của vector chuyển dịch được cực tiểu hóa". Quá trình này cũng chỉ qua 3 lần tính lặp là có kết quả. Cận dưới được xác định để dừng quy trình lặp là sai số do máy đo $( \pm 1 \mathrm{~mm})$. Điều này là phù hợp với dữ liệu đo của chúng ta.

Quá trình so sánh tính thống nhất giữa các chu kỳ quan trắc và quá trình ước lượng điểm ổn định được thực hiện thông qua kiểm định thống kê Fisher theo quy trình nghiêm ngặt. Do khuôn khổ bài báo nên chúng tôi phải giản lược bớt.

Giá trị chuyển dịch được xác định trong quy trình biến đổi trọng số. Sau đó, điểm không ồn định được đánh dâu và tiến hành xây dựng mô hình biến dạng. Đối với công trình thủy điện Tuyên Quang, mô hình biến dạng rất đơn giản, do không có điểm nào được xác định là chuyển dịch.

Quy trình phân tích điểm ổn định có thể kết thúc sau khi mô hình biến dạng được thông qua bằng việc xác định các tín hiệu chuyển dịch thông qua kiểm định thống kê toán học.

\section{Kết luận}

Bài báo đã thực hiện bình sai lưới cơ sở mặt bằng thủy điện Tuyên Quang thông qua thuật toán Mittermayer, sau đó phân tích sự ổn định của điểm lưới bằng phương pháp "Biến đổi tuần tự trọng số". Kết quả cho thấy:

- Giá trị chuyển dịch nhỏ hơn so với chỉ thực hiện bình sai lưới tự do (2,2 mm so với 6,2 mm).

- Vị trí điểm chuyển dịch thay đổi so với chỉ thực hiện bình sai lưới tự do (QT5 thay cho QT3).

- Trọng tâm lưới không thay đổi sau mỗi chu kỳ đo do thực hiện "biến đổi trọng số" nên việc đánh giá chuyển dịch được thuận tiện.

Việc đánh giá vị trí điểm ổn định được so sánh thống nhất giữa các chu kỳ và không dựa trên tiêu chuẩn $q \approx \pm 1 \mathrm{~mm}$. Tiêu chuẩn sai số đo $( \pm 1 \mathrm{~mm})$ dùng trong quá trình tính lặp ma trận trọng số $\mathrm{W}$ phản ánh đúng bản chất bài toán tìm trọng tâm lưới tự do thông qua các trị đo trắc địa.

Sau đây là quy trình phân tích biến dạng của đập thủy điện Tuyên Quang. 


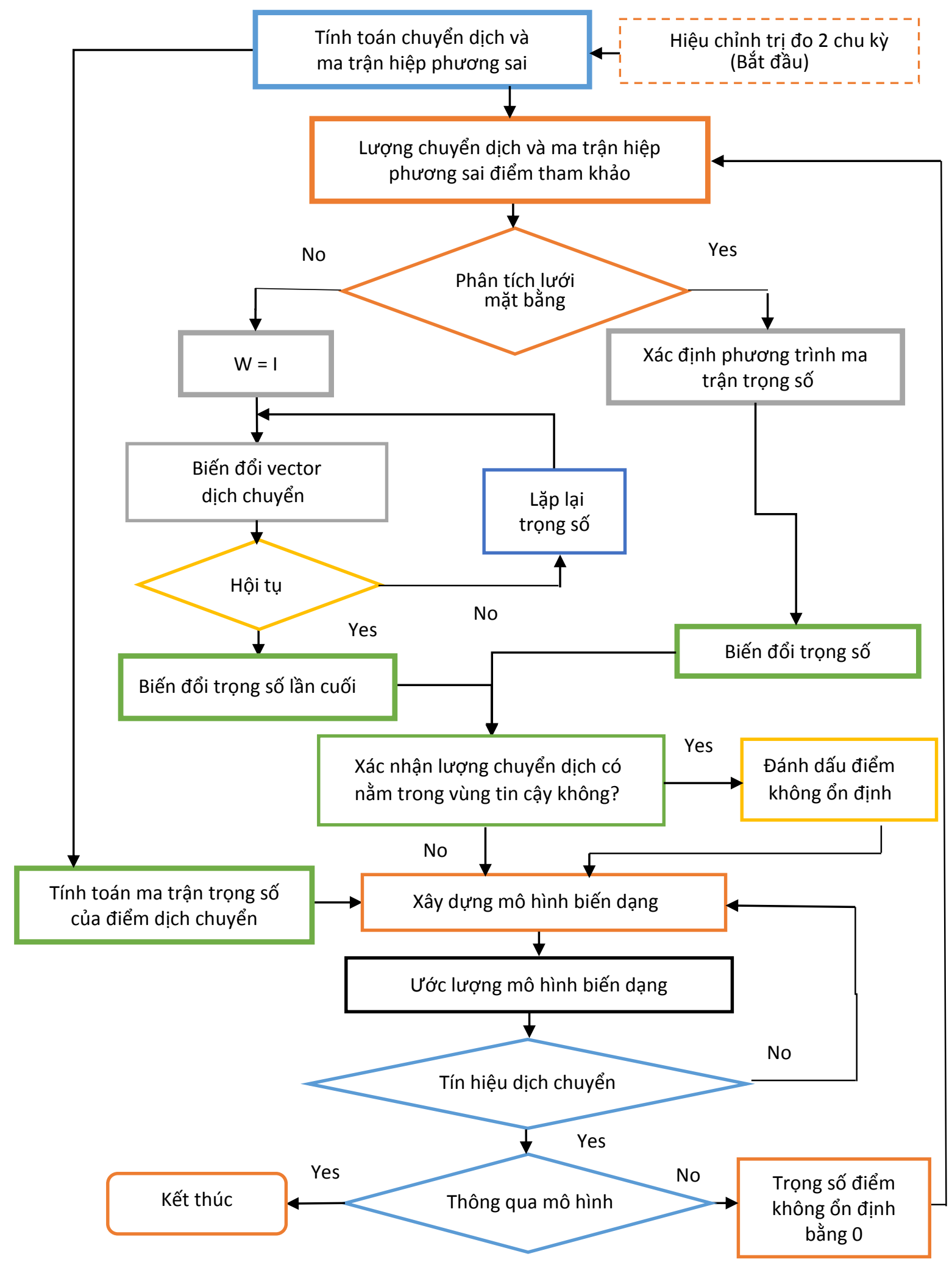




\section{Lời cảm ơn}

Tác giả cảm ơn sự hỗ trợ số liệu quan trắc thủy điện Tuyên Quang của Công ty Cổ phần Tư vấn Xây dựng Điện I. Tác giả cũng cảm ơn những ý kiến đóng góp của người phản biện đã giúp hoàn thiện nội dung bài báo này.

\section{Tài liệu tham khảo}

[1] Đinh Xuân Vinh, Phan Văn Hiến, Nguyễn Bá Dũng, Lý thuyết và phương pháp phân tích biến dạng. Giáo trình đào tạo thạc sĩ, NXB Tài nguyên - Môi trường và Bản đồ Việt Nam, Hà Nội, 2016.

[2] Huang Sheng Xiang, Yin Hui, Jiang Zheng. Phan Văn Hiến biên dịch, Xử lý số liệu quan trắc biến dạng, NXB Khoa học và Kỹ thuật, Hà Nội, 2012.

[3] Adam Chrzanowski, Chen Yongqi, James Michael Secord. Geometrical Analysis of Deformation Surveys. Papers of the Deformation Measurements Workshop, Boston, 31 October- 1 November 1986, p. 369-383. http://www2.unb. ca/ccge/publications/downloads/CCGE\%20\%201986\%20-\%20Geometrical\%20analysis\% 20of\%20deformation\%20surveys.pdf

[4] Phan Văn Hiến, Đinh Xuân Vinh, Phạm Quốc Khánh, Tạ Thanh Loan, Lưu Anh Tuấn, Lý thuyết sai số và bình sai trắc địa, $\mathrm{NXB}$ Xây dựng, Hà Nội, 2017.

[5] Tao Benzao, Phan Văn Hiến biên dịch, Bình sai lưới tự do và phân tích biến dạng, NXB Tài nguyên-Môi trường và Bản đồ Việt Nam, Hà Nội, 2017.
[6] E. Mittermayer, A generalisation of the leastsquares method for the adjustment of free networks. Springer, Bulletin Géodésique (19461975). June 1972, Volume 104, Issue 1, pp 139 157. https://doi.org/10.1007/BF02530298

[7] E.J. Schlossmacher, An iterative technique for absolute deviations curve fitting. Journal of the American Statistical Association. 1973, Vol 68, Issue 344, 857-859. https://doi.org/10.1080/ 01621459.1973 .10481436

[8] Calyampudi Radhakrishna Rao, Sujit Kumar Mitra. Generalized Inverse of Matrices and its Application. Wiley and Sons, New York, 1971. https://archive.org/details/in.ernet.dli.2015.13466 9

[9] Adam Chrzanowski, Chen Yongqi, Analysis of Deformation Surveys - A Generalized method, Technical Report No 94. Department of Geodesy and Geomatics Engineering. University of New Brunswick, P.O. Box 4400, Fredericton, N.B. Canada. E3B 5A3. 1983.

[10] M. Walter Welsch, Otto Heunecke, Models and terminology for the analysis of geodetic monitoring observations. Official Report of the Ad-Hoc Committee of FIG Working Group 6.1, Published by The International Federation of Surveyors (FIG). 2001. Frederiksberg, Denmark. http://fig.net/resources/publications/figpub/pub2 5/figpub25.asp

[11] Công ty Cổ phần Tư vấn Xây dựng Điện I, Số liệu quan trắc điểm cơ sở trắc địa thủy điện Tuyên Quang.

[12] Peter J.Huber, Elvezio M.Ronchetti. Robust statistics, second edition. Published by John Wiley \& Sons, Inc., Hoboken, New Jersey, Canada, 2009. 